cefotaxime was begun. Serum markers for other infectious diseases sometimes complicated by cerebellar ataxia were negative; these included herpes simplex, cytomegalovirus, varicella zoster, mumps, rubella, rubeola, Epstein-Barr virus, and mycoplasma. At discharge on day 28, the neurologic exam was normal, and serum for B burgdorferi IgM and IgG antibodies was positive. (Erol I, Saygi S, Alehan F. Acute cerebellar ataxia in a pediatric case of Lyme disease and a review. Pediatr Neurol 2013 May;48(5):407-10). (Resp: Dr Erol, Adana, Turkey. E-mail: ilknur_erol@yahoo.com).

COMMENT. Neuroborreliosis presents with both central and peripheral nervous system manifestations, including aseptic meningitis, meningoencephalitis, Bell's palsy, radiculoneuritis, and myelitis. Four previously published reports of cerebellar ataxia with Lyme disease are reviewed.

\title{
MRI AS ADJUNCT IN DIAGNOSIS OF MENINGITIS
}

Investigators from the Children's Hospital of Pittsburgh, PA, reviewed the literature on the role of MRI as an adjunct for diagnosing meningitis. Of 7 relevant articles, two were reviews and an opinion of usefulness of the MRI was based on 5 articles. Specificity of MRI (i.e. negative imaging findings in those who did not have meningitis) was high and ranged from $93 \%$ to $100 \%$. Sensitivity of the MRI was more variable $(9 \%, 85 \%, 95 \%$ and $100 \%)$; sensitivity may be higher for bacterial and fungal meningitis than for viral meningitis, but it may depend on the degree of inflammatory response and may vary with etiology. The MRI sequences may vary in yield, the contrastenhanced FLAIR being most useful in a number of studies. Most of the studies included children, but the majority involved adults.

Based on the studies reviewed, MRI is not recommended to rule out meningitis due to its poor sensitivity; it may be more useful for bacterial compared to viral meningitis, but sensitivity varies depending on MRI technique used and data are limited. MRI is more specific but cannot be recommended to rule in meningitis because data are limited for children and none for infants. (Upadhyayula S. Is there a role for MRI as an adjunct for diagnosing bacterial meningitis? Arch Dis Child 2013 May;98(5):388-90). (Response: Dr Shankar Upadhyayula, Infectious Diseases, Children's Hospital of Pittsburgh, PA 15206. E-mail: Shankar.upadhyayula@chp.edu).

COMMENT. If further studies provide a more definitive role, MRI could be of diagnostic value in neonates and small children with traumatic lumbar punctures, to avoid unnecessary long-term antibiotics and extended hospital stay.

\section{NEUROCUTANEOUS DISORDERS}

\section{GM1 GANGLIOSIDOSIS TYPE 1 AND MONGOLIAN SPOTS}

Investigators in Sao Paulo, Brazil, report a female infant born at term to healthy consanguineous parents who was examined at 9 months for delayed development. She showed hepatosplenomegaly, and widespread Mongolian spots extending over the back 
and upper and lower extremities. Funduscopic examination revealed cherry red spot of the retina, consistent with a diagnosis of the lysosomal disease, GM1 gangliosidosis type 1. (Hackbart BA, Arita JH, Pinho RS, Masruha MR, Vilanova LCP. Mongolian spots are not always a benign sign. J Pediatr 2013 May;162(5):1070). (Response: Dr Barbara A Hackbart, Division of Child Neurology, Federal University of Sao Paulo, Brazil).

COMMENT. The Mongolian spots result from entrapment of melanocytes in the dermis because of arrested transdermal migration from the neural crest into the epidermis. Hurler syndrome and GM1 gangliosidosis type 1 are diseases associated with generalized Mongolian spots. Infants with GM1 gangliosidosis type 1, also known as Pseudo-Hurler's disease, show facial abnormalities that include frontal bossing, depressed nasal bridge, macroglossia, large low-set ears, and marked hirsutism. About $50 \%$ have cherry-red spots. The association of Mongolian spots with the lysosomal disease GM1 gangliosidosis type 1 was not recorded in older neurology textbooks, but a PubMed search found 10 references in the last 30 years (Weissbluth $\mathbf{M}$, et al. Br J Dermatol 1981 Feb;104(2):195-200) (Ashrafi MR, et al. Pediatr Neurol 2006 Feb;34(2):143-5). Mongolian spots when unusually numerous should prompt an examination for the lysosomal disease, GM1 gangliosidosis type 1.

\section{DEMYELINATING DISEASES}

\section{DIAGNOSTIC CRITERIA FOR PEDIATRIC MS}

Investigators at Northwestern University Feinberg School of Medicine and Ann \& Robert H. Lurie Children's Hospital of Chicago review the diagnostic criteria for pediatric multiple sclerosis, the differential diagnosis, the $2010 \mathrm{McD}$ onald criteria, and Callen criteria. Of all persons with MS, $2 \%$ to $5 \%$ have onset before 16 years of age. The diagnosis is clinical, requiring recurrent episodes of CNS demyelination, serial changes in MRI lesions, and CSF oligoclonal bands or elevated IgG index. MS must be differentiated from ADEM, neuromyelitis optica and other inflammatory, infectious or metabolic conditions. These include mitochondrial disorders, leukodystrophy, Alexander's disease, MELAS, Kearn-Sayre syndrome, Behcet and Sjogren syndromes, sarcoidosis, Hashimoto's encephalitis, HIV, herpes virus, neuroborreliosis, mycoplasma, the arteriopathy CADASIL, and CNS vasculitis. (Rubin JP, Kuntz NL. Diagnostic criteria for pediatric multiple sclerosis. Curr Neurol Neurosci Rep [Section Editor, Nordli DR Jr] 2013 Jun;13(6):354).

COMMENT. In this excellent and comprehensive review, the differentiation of MS from ADEM and other inflammatory or infectious conditions is stressed. Transient demyelinating events must be distinguished from a life-long diagnosis of MS.

Cerebral venous thrombosis (CVT) after LP and steroids in childhood MS. The association between CVT and MS is reported in a 13-year-old girl admitted with left hemiparesis, ataxia, and headache following vaccination against meningococcal group $\mathrm{C}$ and hepatitis A. LP and high dose corticosteroids for MS may have contributed to the CVT (Presicci A, et al. Brain Dev 2013 Jun;35(6):602-5). 